\title{
A Framework for Estimating the Eligible Patient Population for New Migraine Acute Therapies in the United States
}

\author{
Linda Harris · Gilbert L'Italien • Thomas O'Connell (D) • \\ Zacharia Hasan · Susan Hutchinson · Sylvia Lucas
}

Received: February 8, 2021 / Accepted: May 7, 2021 / Published online: May 31, 2021

(C) The Author(s) 2021, corrected publication [2021]

\section{ABSTRACT}

Introduction: Migraine is associated with considerable disability for patients not adequately managed with current standards of care. New acute therapies may offer relief for this population of patients; however, population size and associated potential costs of new therapies are unclear. In this study, a conceptual framework was developed to estimate anticipated use of new acute therapies.

Methods: Targeted literature review (TLR) was conducted to identify factors affecting access to migraine-specific acute therapies, and characteristics of individuals who would be eligible for new acute therapies. Findings from the TLR were combined to create a framework for

Supplementary Information The online version contains supplementary material available at https:// doi.org/10.1007/s12325-021-01781-z.

L. Harris · G. L'Italien

Biohaven Pharmaceuticals, New Haven, CT, USA

T. O'Connell $(\bowtie) \cdot$ Z. Hasan

Medicus Economics, Boston, MA, USA

e-mail: thomas.oconnell@gmail.com

S. Hutchinson

Orange County Migraine and Headache Center, Irvine, CA, USA

S. Lucas

Department of Neurology, University of

Washington Medical Center, Seattle, WA, USA estimating the size of the eligible patient population. This framework was used to calculate two estimates of the eligible patient population by applying parameters (i) identified in the TLR and (ii) from a recent budget-impact analysis (BIA).

Results: The primary factors affecting access to migraine-specific acute therapies identified in the TLR were consulting a healthcare professional for headache, receiving a migraine diagnosis, and receiving a prescription for migrainespecific treatment. Characteristics of individuals likely to use new acute therapies reflected in the TLR were contraindication to triptans, or failure to respond to/tolerate at least two oral triptans. Application of the framework suggested that $15-25 \%$ of individuals with migraine would be eligible for new acute therapies.

Conclusion: A limited number of patients currently use migraine-specific acute therapies. Among such patients, a significant proportion do not have adequate symptom control. Accordingly, a minority of individuals with migraine may be expected to use new acute therapies. The framework developed in this study is intended to facilitate estimating the eligible patient population in assessments of costs of new acute therapies. Such assessments should also consider recommendations that patients have access to multiple types of acute therapies, which may yield savings from reduced medication-overuse headache $(\mathrm{MOH})$, progression to chronic migraine, and urgent- 
care costs.Keywords: Migraine, acute; CGRP; 5$\mathrm{HT}_{1 \mathrm{~F}}$; Rimegepant; Ubrogepant; Lasmiditan; Triptan; Eligibility; Budget impact; Model

\section{Key Summary Points}

Why carry out this study?

Migraine is a common condition affecting approximately 30 million adults and 9 million children and adolescents in the US; symptoms and disability during an attack are managed with acute treatments, including simple analgesics, nonsteroidal anti-inflammatory drugs (NSAIDs), opioid analgesics, butalbital-containing analgesic products, and over-the-counter combinations of analgesics and caffeine, as well as prescription migraine-specific agents which historically have included triptans and ergot derivatives (e.g., dihydroergotamine).

Triptans are commonly used for acute treatment of migraine attacks. However, some patients may not be adequately managed with triptans because of lack of efficacy; intolerable side effects known as "triptan sensations" (including nausea, fatigue, malaise, rapid heart rate, feelings of tingling, numbness, warmth, and chest/neck pressure or tightness); and safety concerns for those with a history of vascular disease, multiple risk factors for vascular disease (such as hypertension and diabetes), and during pregnancy.

The US Food and Drug Administration (FDA) has recently approved three new acute treatments for migrainerimegepant (NURTEC ${ }^{\mathrm{TM}}$ ODT) and ubrogepant (UBRELVY ${ }^{\circledR}$ ), both CGRP receptor antagonists, and lasmiditan $\left(\right.$ REYVOW $\left.^{\circledR}\right)$, a $5-\mathrm{HT}_{1 \mathrm{~F}}$ receptor agonistwhich the American Headache Society (AHS) has recommended for patients who have contraindications to triptans or who have failed to respond to or tolerate at least two oral triptans.
Understanding the size of the patient population likely to use new treatments may be of interest to payers and health systems; in this study, we therefore developed a conceptual framework for estimating anticipated use of new acute therapies, based on a targeted literature review (TLR) and insights from clinical experience.

\section{What was learned from the study?}

A minority of individuals with migraine (15\%-25\%) may be expected to use new acute therapies, given that only a limited proportion of patients currently use migraine-specific acute therapies. Among such patients, a significant proportion do not have adequate symptom control.

The framework developed in this study is intended to facilitate estimating the eligible patient population in assessments of costs of new acute therapies. Such assessments should also consider recommendations that patients have access to multiple types of acute therapies, which may yield savings from reduced $\mathrm{MOH}$, progression to chronic migraine, and urgent-care costs.

\section{DIGITAL FEATURES}

This article is published with digital features, including a summary slide to facilitate understanding of the article. To view digital features for this article go to https://doi.org/10.6084/ m9.figshare.14495814.

\section{INTRODUCTION}

Migraine is a neurological condition that manifests as recurrent attacks of headache lasting approximately $4-72 \mathrm{~h}$ [1]. The headache may be unilateral and have a pulsating or throbbing quality, is generally worsened by physical activity, and may be associated with nausea/ vomiting and/or sensitivity to light and sound 
[1]. If unsuccessfully treated, headache severity may progress throughout an attack. Migraine is classified on the basis of monthly frequency of headache days as either episodic (1-14 monthly headache days, MHDs) or chronic (at least 15 MHDs, with features of migraine headache on at least 8 days per month [2]) [1]. In the 2016 Global Burden of Disease Study, migraine was the leading cause of years lived with disability globally in patients younger than 50 years of age, and second only to lower back pain in all patients [3].

Treatments for migraine are typically distinguished as "acute" or "preventive". Acute treatment is intended to alleviate migraine pain and associated symptoms and to restore ability to function during a migraine attack, while preventive treatment aims to reduce attack frequency, severity, duration, and disability and may improve response to acute therapies [4]. Acute treatments used to treat mild-to-moderate headache include simple analgesics (e.g., aspirin, acetaminophen), nonsteroidal anti-inflammatory drugs (NSAIDs, e.g., ibuprofen, naproxen), opioid analgesics, butalbital-containing analgesic products, and over-the-counter combinations of analgesics including caffeine (e.g., aspirin + acetaminophen + caffeine) $[4,5]$. Following diagnosis of migraine, migraine-specific agents may be prescribed for acute treatment, including triptans and ergot derivatives. While effective acute treatment may alleviate symptoms and reduce disability for patients, suboptimal acute treatment may lead to an increase in headache frequency and disability [4]. In particular, overuse of acute treatments is associated with medication-overuse headache $(\mathrm{MOH})$ [6], and increased headache frequency is associated with increased risk of progression from episodic to chronic migraine [7]. Treatment guidelines stress that successful management of an attack is most likely if acute therapy is taken early, and if patients understand the condition and have a treatment strategy $[4,5]$.

The US Food and Drug Administration (FDA) has recently approved several new oral therapies for acute treatment of migraine, including rimegepant (NURTEC ${ }^{\mathrm{TM}}$ ODT, Biohaven Pharmaceuticals) and ubrogepant (UBRELVY ${ }^{\circledR}$,
Allergan), both CGRP receptor antagonists, as well as lasmiditan (REYVOW $^{\circledR}$, Eli Lilly and Company), a 5- $\mathrm{HT}_{1 \mathrm{~F}}$ receptor agonist (and first in its class of the "ditans"). While triptans may provide meaningful relief, patients who experience inadequate relief may "cycle" through the multiple formulations of triptans (seven triptans are available, some with multiple routes of administration [5]), and may use more than the recommended number of doses per month and risk $\mathrm{MOH}$ [5]. Some patients may not tolerate side effects of triptans, known as "triptan sensations", which may include nausea, fatigue, malaise, rapid heart rate, feelings of tingling, numbness, warmth, and pressure or tightness in the chest or neck [8]. Other patients may not be candidates for the triptans. As $5-\mathrm{HT}_{1 \mathrm{~B} / 1 \mathrm{D}}$ agonists, vasoconstriction (attributed to their 1B agonist effects) potentially makes them unsafe for patients with history of vascular disease (either cardiovascular, cerebrovascular, or peripheral vascular disease) or with multiple risk factors for vascular disease. Recently, the American Headache Society (AHS) indicated that new acute treatments should be considered in patients who have contraindications to triptans or who have failed to respond to or tolerate at least two oral triptans [4].

Migraine is a common condition, estimated to affect approximately 30 million adults in the US ( $15 \%$ of the adult population), as well as 9 million children and adolescents. Understanding the size of the patient population likely to use new treatments may therefore be of significant interest to payers and health systems [9]. In this study, we developed a conceptual framework for estimating anticipated use of new acute therapies, based on a targeted literature review (TLR) and insights from clinical experience. The framework identifies factors affecting access to migraine-specific (i.e., prescription) acute therapies and the characteristics of patients who would use new acute therapies. We then illustrated use of the framework by estimating the size of the patient population eligible for new migraine-specific acute therapies in the US, applying parameter values (i) identified in the TLR and (ii) modeled in a recent budget-impact analysis (BIA) of new acute therapies conducted by the Institute for 
Clinical and Economic Review (ICER). Finally, considerations for modeling the costs of new acute treatments for migraine, beyond those for estimating the eligible patient population, are discussed on the basis of results of the TLR.

\section{METHODS}

The TLR was conducted using the MEDLINE database to identify literature published from 2000 to 2021 on real-world use of migrainespecific (i.e., prescription) acute therapies. Animal studies were excluded, and articles were limited to those published in English and with an abstract. Results of the search were then screened on the basis of title to restrict to realworld studies of acute-therapy use and acutetherapy recommendations/guidelines. Types of results excluded in the screening step included descriptive publications (e.g., reporting on symptoms and complications, pathophysiology, patient-reported outcome tools, and biological mechanisms of side effects of treatments), prospective studies of efficacy of migraine therapies (including acute and preventive), cost-effectiveness analyses, and other studies of non-acute therapies (including nonmigraine specific and preventive). Eligibility of remaining publications was confirmed on the basis of review of abstracts; publications were excluded if their abstract suggested they were review articles of efficacy and safety results or studies of non-US populations. A publication's full text was reviewed to confirm eligibility when deemed necessary by the reviewers (TO and $\mathrm{ZH}$ ). Following confirmation of eligibility and review of the publications, their findings were summarized by the reviewers and discussed among all authors of this study. Two group discussions were held to review findings of the TLR.

The conceptual framework was developed by relating findings of the TLR and group discussions to the American Headache Society (AHS) recommendation that new acute treatments should be considered in patients who have contraindications to triptans or who have failed to respond to or tolerate at least two oral triptans [4]. Specifically, to estimate the patient population eligible for new acute therapies, factors affecting access to migraine-specific acute therapies were first specified. Then, among individuals who would access migrainespecific acute therapies, characteristics of those who would be eligible for new acute therapies were further specified. For illustration of use of the framework, two estimates of the eligible patient population were calculated by applying parameters (i) identified in the TLR and (ii) from the BIA released on February 25, 2020 in ICER's Final Evidence Report on treatments for "acute migraine" [10].

This analysis was based on previously conducted studies and does not contain any new studies with human participants or animals performed by any of the authors.

\section{RESULTS}

The TLR included 236 results meeting the search terms and restrictions, 56 of which were retained after screening and eligibility reviews, as illustrated in the PRISMA (Preferred Reporting Items for Systematic Reviews and MetaAnalyses) flow diagram in Fig. 1.

Findings of publications retrieved in the TLR revealed several prerequisites for patient access to migraine-specific acute therapies. Real-world studies suggest that in the US, individuals meeting diagnostic criteria for migraine may not consult a healthcare professional routinely (only $45.5 \%$ sought care for their headache in the last year [11] and $79.8 \%$ in their lifetime [12]). As described by Lipton et al. [11], the consulting rate is significantly higher in those with insurance coverage vs. those without. Among individuals who do consult a healthcare professional for acute headache, evidence indicates that the majority meet diagnostic criteria for migraine (84\% in outpatient clinics [13] and $95 \%$ in the emergency department (ED) [14]); nonetheless, a diagnosis of migraine may not be reached. In US studies, the diagnosis rate of migraine among patients meeting diagnostic criteria varies considerably, from $45.7 \%$ in the Migraine in America Symptoms and Treatment (MAST) study [12] to $86.7 \%$ in the American Migraine Prevalence and Prevention (AMPP) 


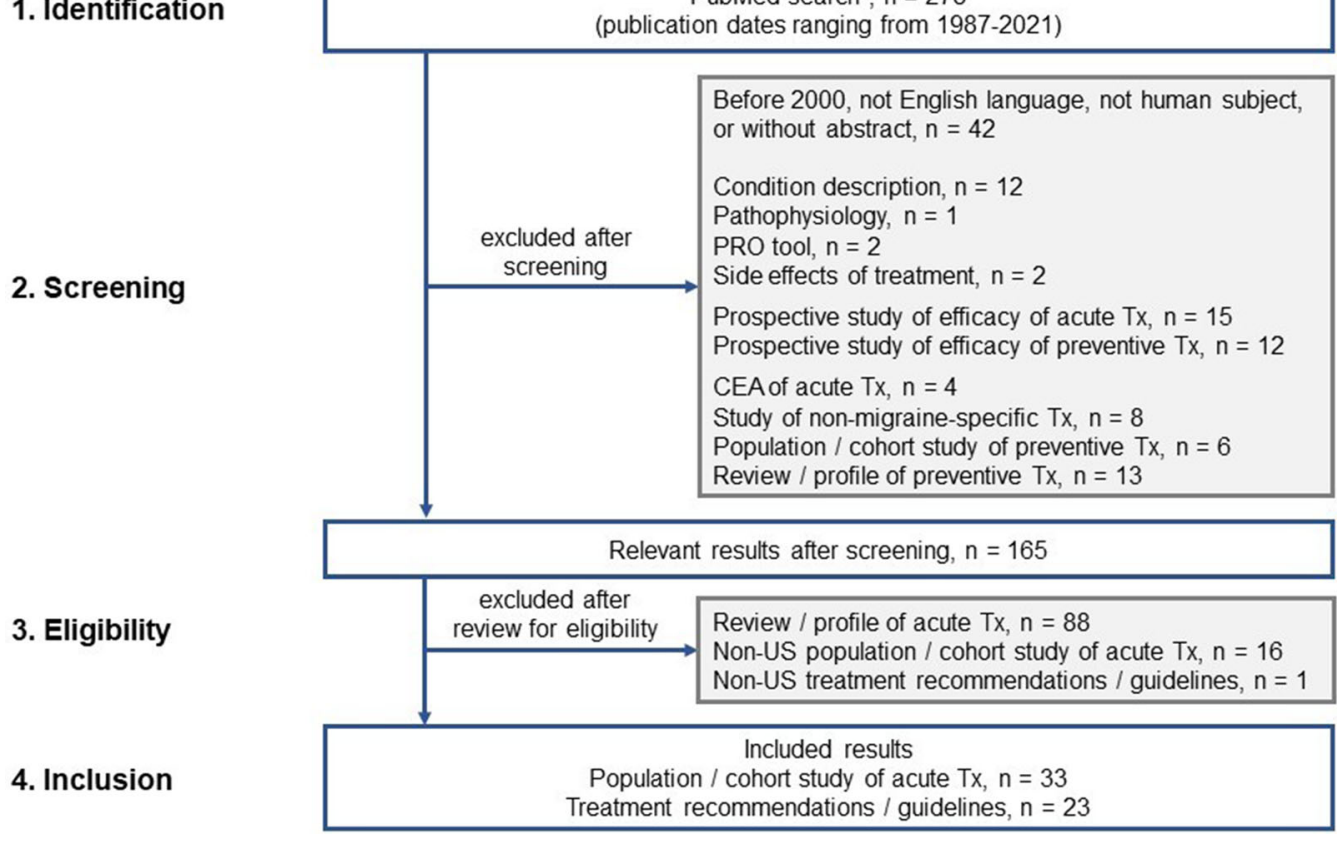

Fig. 1 PRISMA flow diagram of excluded and included publications in the TLR. Abbreviations: CEA cost-effectiveness analysis, PRO patient-reported outcome, RCT randomized controlled trial, TLR targeted literature review, Tx treatment. Note: The PubMed search was most recently conducted on March 23, 2021, using

study [11]. Reaching a diagnosis is particularly challenging in the ED setting [14] as conditions such as anxiety and depression are commonly comorbid with migraine and may confound the cause of a patient's symptoms [15]. Among consulting patients, women appear to be more likely to receive a migraine diagnosis than men $[11,12]$.

Among patients diagnosed with migraine and who can therefore be prescribed migrainespecific acute therapies, triptans are commonly recommended as first-line options [4, 5]. However, not all patients are successfully managed with triptans for several reasons, including lack of efficacy and intolerable side effects. In the US, seven types of triptans are available [5], several of which have multiple routes of administration (oral, oral disintegrating tablet, nasal spray, injection) and may be available as generic or branded products [5]. Evidence suggests that determining the triptan formulation
Medical Subject Headings (MeSH) Major Topic and title/abstract keywords targeting acute, prescription therapies: ("migraine disorders/drug therapy" [MeSH Major Topic]) AND (acute[Title/Abstract]) AND ((specific[Title/Abstract]) OR (prescription[Title/Abstract]))

with optimal efficacy for a certain patient may therefore require trial of multiple triptans ("cycling") $[16,17]$. However, ability to tolerate the trial of multiple triptans may be limited given their potential known side effects ("triptan sensations"). Further, a significant proportion of patients who might benefit from migrainespecific acute therapy may not be candidates for use of triptans. In patients with vascular disease or with multiple risk factors for vascular disease (e.g., advanced age, hypertension, smoking, obesity, diabetes, family history of coronary artery disease), vasoconstriction attributed to triptans' $1 \mathrm{~B}$ agonist effects poses safety concerns $[6,18-20]$. In addition, during pregnancy, the benefit of triptans should be weighed against their risk (i.e., they are deemed FDA Category $\mathrm{C}$ - see note in supplementary information) $[6,21]$.

The aforementioned potential limitations of triptans likely contribute to their relatively low 
use observed in real-world studies, estimated at $20-30 \%$ of individuals meeting diagnostic criteria for migraine (including ca. 20\% in the AMPP study [22], 23\% in the Chronic Migraine Epidemiology and Outcomes (CaMEO) study [23], 26\% in the MAST study [24], and 29\% in the Medical Expenditure Panel Survey (MEPS) [25]). Beyond current users of triptans, a significant proportion of patients may have discontinued use; in the CaMEO study, compared to the $23 \%$ currently using migraine-specific acute therapy, $13 \%$ were former users, among whom only one in five reported being able to work or function normally with a headache [26]. Consistent with the CaMEO study, in a recent study of eligibility for acute treatments using a web-based survey of a representative US sample [27], $18.2 \%$ of individuals meeting diagnostic criteria had contraindications to triptans, and $8.6 \%$ reported prior use of triptans but did not use them currently [27]. Among patients currently using triptans, approximately half experienced "poor" or "very poor" efficacy according to the migraine Treatment Optimization Questionnaire (mTOQ) $(\mathrm{mTOQ}<6)$ [27].

Figure 2 presents the conceptual framework developed, the steps of which include (1) consulting a healthcare professional for headache, (2) receiving a migraine diagnosis, (3) receiving a prescription for acute migraine-specific treatment, and (4) being inadequately managed on triptans because of lack of efficacy, intolerance, or safety concerns.

The conceptual framework was applied using parameter values identified in the TLR, which yielded an estimate that $21.6 \%$ of prevalent migraine patients might be eligible for new acute therapies. Specifically, the AMPP study reports that in respondents meeting diagnostic criteria for migraine, $45.5 \%$ consulted a healthcare professional, of whom $86.7 \%$ received a medical diagnosis of migraine, and $66.7 \%$ of those diagnosed currently used migraine-specific acute therapies [11]. These parameter values were applied in steps $1-3$ of the conceptual framework, as reflected in Fig. 2. In step 4, the percentage of migraine-specific acute therapy users inadequately managed with triptans was modeled on the basis of the
OVERCOME (ObserVational survey of the Epidemiology, tReatment, and Care Of MigrainE) study [27], due to its recency and distinction of causes of inadequate management with triptans (i.e., lack of efficacy, intolerance, or contraindication). As described in notes to Fig. 2, OVERCOME found that among the prevalent migraine population, $18.2 \%$ had contraindications to triptans, and among those with history of use of at least one triptan for acute treatment, patients reported inadequate efficacy or discontinuation of triptans twice as often as satisfactory efficacy. In particular, on the basis of the migraine Treatment Optimization Questionnaire (mTOQ), triptan users who experienced "poor" or "very poor" efficacy $(\mathrm{mTOQ}<6)$ or discontinued were approximately double in number vs. those who experienced "moderate" to "maximum" efficacy (mTOQ $\geq 6$ ).

The conceptual framework was also applied using parameter values reported in the ICER BIA, which yielded an estimate that $17.1 \%$ of prevalent migraine patients might be eligible for new acute therapies. In the ICER BIA, two parameters were used to estimate the proportion of prevalent patients eligible for new acute treatments. First, a percentage of prevalent patients using prescription acute treatments was applied. On the basis of the AMPP, ICER modeled that $48.9 \%$ of prevalent patients use prescription migraine medications. Second, a percentage of prescription-medication users for whom triptans would not be effective or tolerated was applied. ICER modeled this at 35\%, based on an estimate that triptans are effective in $60-70 \%$ of migraine patients who have used them [28].

Use of the conceptual framework developed in this study thus illustrated that $15-25 \%$ of prevalent migraine patients might be eligible for new acute treatments, as presented in Fig. 2. While estimates based on the two sets of population mean parameter values considered were similar, results of the TLR suggest that they may be sensitive to characteristics of the patient population considered (e.g., see Table 1 of Lipton et al. [11]) and nature of the setting of care (e.g., lower diagnosis rates in the ED [14]). 


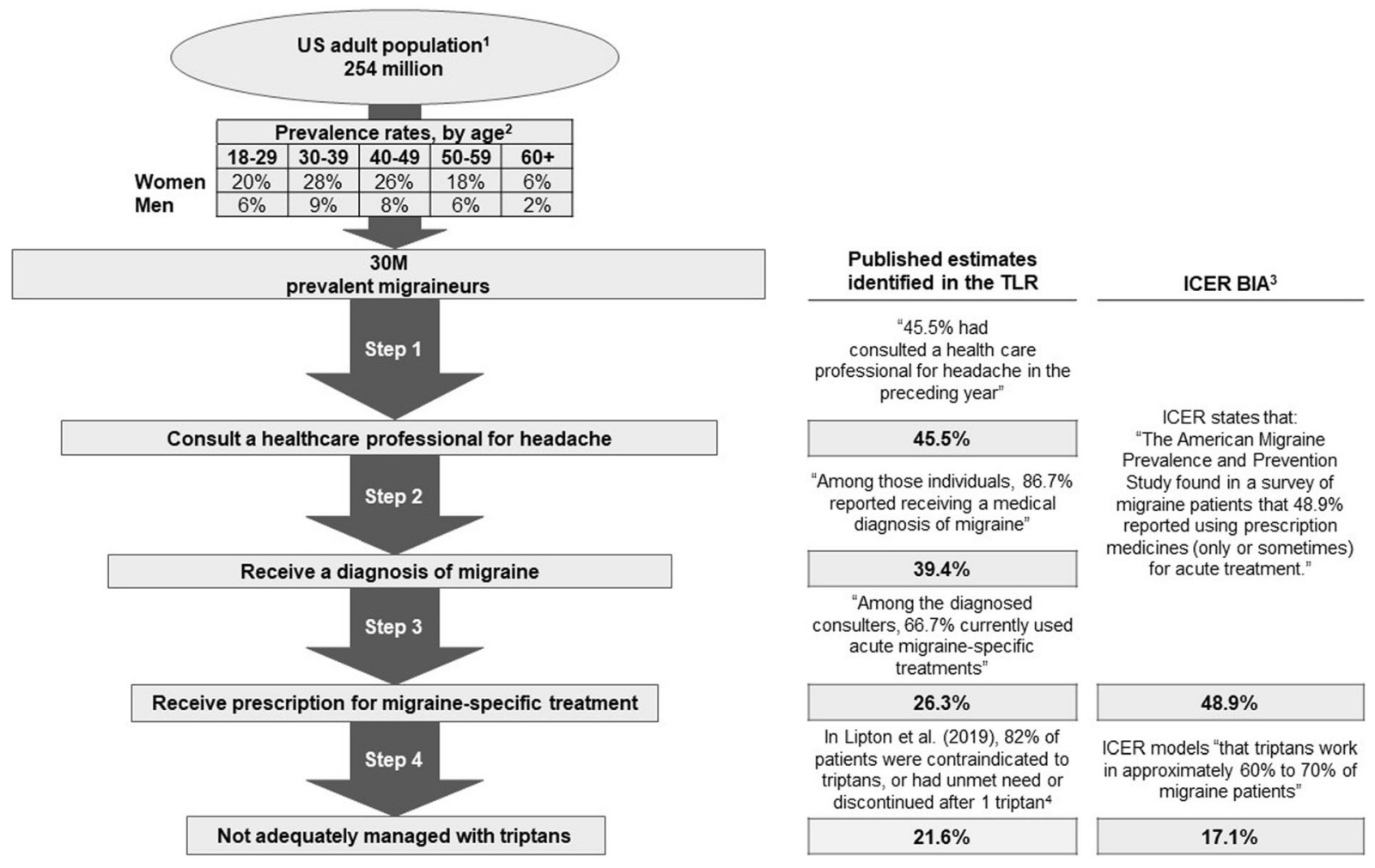

Fig. 2 Framework for estimating the patient population eligible for new acute therapies in the US, and illustration of application of parameter values from the TLR and ICER BIA. Abbreviations: AMS American Migraine Study, BIA budget impact analysis, ICER Institute for Clinical and Economic Review, IHS International Headache Society, MTOQ Migraine Treatment Optimization Questionnaire, TLR targeted literature review, United States of America. Notes: 1. Calculated on the basis of US Census Bureau estimates [48] of population aged 18 or older in 2018. Note that for calculation of prevalent migraine patients, prevalence rates are applied to population totals in each age/sex group. 2. Prevalence rates as reported in Table 2 of Lipton et al. [49]. 3. Reflects the budget impact analysis described in section 7 ("Potential

Budget Impact") of ICER's February 25, 2020 Final Evidence Report [10]. 4. Lipton et al. [27] report that in the OVERCOME web-based survey, of all respondents, the population eligible for novel acute treatments consists of (i) $7.9 \%$ who are not contraindicated, currently on an oral triptan with MTOQ $>6$ (moderate to maximum efficacy), (ii) $8.2 \%$ who are not contraindicated, currently on an oral triptan with MTOQ $\leq 6$ (poor to very poor efficacy), (iii) $8.6 \%$ who are not contraindicated, not currently on an oral triptan but with prior history, and (iv) $18.2 \%$ who are contraindicated. This suggests that of those who may require migraine-specific treatment, ca. $82 \%$ (i.e., $(8.2 \%+8.6 \%+18.2 \%) /(7.9 \%+8.2 \%+8.6 \%+$ $18.2 \%)$ ) are not adequately managed on triptans

\section{DISCUSSION}

While the conceptual framework developed in this study provides guidance for estimating the number of patients who may try new acute therapies, assessment of total costs also requires estimating the frequency of use of new therapies and the impact on frequency of use of existing therapies. BIAs, including the ICER BIA

considered in this report, often assume that eligible patients would use a new therapy rather than an alternative-e.g., that patients would use rimegepant, ubrogepant, or lasmiditan instead of a triptan. However, in acute treatment for migraine, such a substitution may not occur in practice. Some management strategies suggest using a stratified approach [5, 29-33], involving use of different acute therapies 
depending on the severity of a migraine attack, recognizing that the attacks experienced by a single patient may vary considerably in clinical features and severity $[4,34,35]$. Other strategies involve "step care within attacks" [5], whereby a patient would use a simple analgesic initially, but switch to more potent medications later in the same attack, as necessary. Considering that different acute therapies may be needed for effective management in different attacks or within an attack, patients may require access to an array of acute therapies in order to follow a successful management strategy [30, 35-37].

If access to new acute therapies allows patients to follow their recommended management strategy more effectively, assessments of total costs should account for the potential cost offsets associated with improved effectiveness of management [33]. Ineffective acute treatment may lead to $\mathrm{MOH}[4,5]$ or progression to chronic migraine $[7,38]$, which are associated with higher healthcare resource use and costs [39]. In particular, suboptimal acute management of migraine increases the likelihood of an ED visit [40], and annual frequency of ED visits is associated with higher annual healthcare costs (\$3125 in incremental annual costs associated with at least two annual ED visits [41]). In migraine patients, opioids are most commonly prescribed in the ED [42], for patients who have not been able to achieve effective acute management of migraine, and are associated with significantly higher annual healthcare costs (incremental costs of $\$ 1985$ for 1-2 annual prescriptions, $\$ 4107$ for $3-6$, and $\$ 6332$ for at least 7 [41]).

Finally, in assessing the costs of acute treatments for migraine, healthcare decision-makers may benefit from considering patient and physician education initiatives around effective acute treatment strategies. Many patients with migraine have never used migraine-specific acute therapies $[23,43]$ and among those who have, a significant proportion may delay use of acute treatments to a point where they are of lesser effectiveness [44, 45]. Guidelines for acute treatment therefore stress the importance of ensuring that patients understand the condition and their treatment strategy, and that acute therapy be taken early in attacks $[4,5]$.
Education initiatives may therefore help to minimize ineffective use of acute therapies and associated healthcare resource use (e.g., ED visits) $[46,47]$.

\section{CONCLUSIONS}

In this study, we conducted a TLR which was used to inform development of a conceptual framework for estimating the eligible patient population for new acute therapies for migraine in the US. Using the framework, we illustrated that applying population mean parameter values (i) identified in the TLR and (ii) used in ICER's BIA of new acute therapies suggested that $15-25 \%$ of prevalent migraine patients might be eligible for new acute therapies. Beyond estimation of the eligible patient population, other important factors to consider in assessment of total costs of new acute therapies include that effective management strategies call for access to multiple acute therapies, and that ineffective acute treatment is associated both with considerable burden for patients (including risk of $\mathrm{MOH}$ and progression to chronic migraine) as well as significantly higher healthcare resource-use costs. Access to effective acute therapies, for use when necessary, coupled with patient and physician education on recommended management strategies, may significantly impact patient outcomes and costs.

\section{ACKNOWLEDGEMENTS}

Funding. Biohaven Pharmaceuticals, Inc. sponsored the review and development of the manuscript and funded all article processing charges. The authors approved the final version to be published after critically revising the manuscript/publication for important intellectual content. The publication of study results was not contingent on the sponsor's approval or censorship of the manuscript.

Editorial Assistance. The authors would like to acknowledge editorial review provided by 
Kathryn Keller, PharmD (formerly Associate Director, Medical Science Liaison at Biohaven Pharmaceuticals, Inc.).

Authorship. All named authors meet the International Committee of Medical Journal Editors (ICMJE) criteria for authorship for this article, take responsibility for the integrity of the work as a whole, and have given their approval for this version to be published.

Disclosures. Susan Hutchinson has received honoraria or consulting fees from Alder/Lundbeck, Allergan/AbbVie, Amgen, Biohaven, Currax, electroCore, Impel, Lilly, Novartis, Teva, Theranica, and Upsher-Smith. Sylvia Lucas has received honoraria or consulting fees from Alder, Allergan, Amgen, Biohaven, Lilly, Lundbeck, and Teva. Linda Harris and Gilbert L'Italien are employees of, and may own stock/ options in, Biohaven Pharmaceuticals, Inc. At the time of this study, Thomas $\mathrm{O}^{\prime}$ Connell and Zacharia Hasan were employees of Medicus Economics, LLC, a paid consultant of Biohaven Pharmaceuticals, Inc. Biohaven Pharmaceuticals paid the publication charges for this piece, via reimbursement to Medicus Economics.

Compliance with Ethics Guidelines. This article is based on previously conducted studies and does not contain any new studies with human participants or animals performed by any of the authors.

Data Availability. Data sharing is not applicable to this article as no datasets were generated or analyzed during the current study.

Open Access. This article is licensed under a Creative Commons Attribution-NonCommercial 4.0 International License, which permits any non-commercial use, sharing, adaptation, distribution and reproduction in any medium or format, as long as you give appropriate credit to the original author(s) and the source, provide a link to the Creative Commons licence, and indicate if changes were made. The images or other third party material in this article are included in the article's Creative Commons licence, unless indicated otherwise in a credit line to the material. If material is not included in the article's Creative Commons licence and your intended use is not permitted by statutory regulation or exceeds the permitted use, you will need to obtain permission directly from the copyright holder. To view a copy of this licence, visit http://creativecommons.org/licenses/by$\mathrm{nc} / 4.0 /$.

\section{REFERENCES}

1. Edvinsson L, Haanes KA, Warfvinge K, Krause DN. CGRP as the target of new migraine therapies successful translation from bench to clinic. Nat Rev Neurol. 2018;14(6):338-50.

2. Cutrer MF, Bajwa ZH. Pathophysiology, clinical manifestations, and diagnosis of migraine in adults. In: Swanson JW, Dashe JF, editors. UpToDate. Nov 17, 2018. https://www.uptodate.com/contents/ pathophysiology-clinical-manifestations-anddiagnosis-of-migraine-in-adults. Accessed 22 May 2019.

3. GBD 2016 Disease and Injury Incidence and Prevalence Collaborators. Global, regional, and national incidence, prevalence, and years lived with disability for 328 diseases and injuries for 195 countries, 1990-2016: a systematic analysis for the Global Burden of Disease Study 2016. Lancet. 2017;390(10100):1211-59.

4. American Headache Society. The American Headache Society position statement on integrating new migraine treatments into clinical practice. Headache. 2019;59(1):1-18.

5. Mayans L, Walling A. Acute migraine headache: treatment strategies. Am Fam Phys. 2018;97(4): 243-51.

6. Bajwa $\mathrm{ZH}$, Smith JH. Acute treatment of migraine in adults. In: Swanson JW, Dashe JF, editors. UpToDate. Mar 11, 2019. https://www.uptodate.com/ contents/acute-treatment-of-migraine-in-adults. Accessed 22 May 2019.

7. Buse DC, Greisman JD, Baigi K, Lipton RB. Migraine progression: a systematic review. Headache. 2019;59(3):306-38.

8. Nappi G, Sandrini G, Sances G. Tolerability of the triptans: clinical implications. Drug Saf. 2003;26(2): 93-107.

9. Joshua Cohen. Migraine breakthrough: not so fast [internet]. Forbes. Jun 6, 2018. https://www.forbes. 
com/sites/joshuacohen/2018/06/06/migraine-

breakthrough-not-so-fast/. Accessed 22 May 2019.

10. Institute for Clinical and Economic Review. Acute treatments for migraine - final evidence report. Feb 25, 2020. https://icer-review.org/wp-content/ uploads/2019/06/ICER_Acute-Migraine_Final-

Evidence-Report_updated_030320.pdf. Accessed 18 May 2020.

11. Lipton RB, Serrano D, Holland S, Fanning KM, Reed ML, Buse DC. Barriers to the diagnosis and treatment of migraine: effects of sex, income, and headache features. Headache. 2013;53(1):81-92.

12. Lipton RB, Munjal S, Alam A, et al. Migraine in America Symptoms and Treatment (MAST) study: baseline study methods, treatment patterns, and gender differences. Headache. 2018;58(9):1408-26.

13. Kaniecki R, Ruoff G, Smith T, et al. Prevalence of migraine and response to sumatriptan in patients self-reporting tension/stress headache. Curr Med Res Opin. 2006;22(8):1535-44.

14. Blumenthal HJ, Weisz MA, Kelly KM, Mayer RL, Blonsky J. Treatment of primary headache in the emergency department. Headache. 2003;43(10): 1026-31.

15. Hutchinson SL, Silberstein SD. Menstrual migraine: case studies of women with estrogen-related headaches. Headache. 2008;48(Suppl 3):S131-41.

16. Buzzi MG. Pathways to the best fit of triptans for migraine patients. Cephalalgia. 2008;28(Suppl 2): 21-7.

17. Diamond ML, Wenzel RG, Nissan GR. Optimizing migraine therapy: evidence-based and patient-centered care. Expert Rev Neurother. 2006;6(6):911-9.

18. Lipton RB, Diamond S, Reed M, Diamond ML, Stewart WF. Migraine diagnosis and treatment: results from the American Migraine Study II. Headache. 2001;41(7):638-45.

19. Martin VT, Goldstein JA. Evaluating the safety and tolerability profile of acute treatments for migraine. Am J Med. 2005;118(Suppl 1):36s-44s.

20. Olesen J, Tfelt-Hansen P, Ashina M. Finding new drug targets for the treatment of migraine attacks. Cephalalgia. 2009;29(9):909-20.

21. Amundsen S, Nordeng H, Nezvalová-Henriksen K, Stovner LJ, Spigset O. Pharmacological treatment of migraine during pregnancy and breastfeeding. Nat Rev Neurol. 2015;11(4):209-19.
22. Bigal ME, Borucho S, Serrano D, Lipton RB. The acute treatment of episodic and chronic migraine in the USA. Cephalalgia. 2009;29(8):891-7.

23. Hutchinson S, Lipton RB, Ailani J, et al. Characterization of acute prescription migraine medication use: results from the CaMEO study. Mayo Clin Proc. 2020;95(4):709-18.

24. Lipton RB, Munjal S, Buse DC, et al. Unmet acute treatment needs from the 2017 migraine in America symptoms and treatment study. Headache. 2019;59(8):1310-23.

25. Wu J, Noxon V, Lu ZK. Patterns of use and health expenses associated with triptans among adults with migraines. Clin J Pain. 2015;31(8):673-9.

26. Litwin AH, Harris KA Jr, Nahvi S, et al. Successful treatment of chronic hepatitis $\mathrm{C}$ with pegylated interferon in combination with ribavirin in a methadone maintenance treatment program. J Subst Abuse Treat. 2009;37(1):32-40.

27. Lipton R, Pohl G, Araujo A, et al. Who is eligible for novel medications designed for the acute treatment of migraine and what are their unmet needs? Results of the OVERCOME study. Presented at 2019 American Headache Society Annual Meeting; July 11-14, 2019; Philadelphia, PA. Abstract 632440.

28. Oliver RL, Taylor A. Treatment-resistant migraines. Jan 5, $2012 . \quad$ https://www. practicalpainmanagement.com/pain/headache/ migraine/treatment-resistant-migraines. Accessed 18 May 2020.

29. Bigal ME, Lipton RB, Krymchantowski AV. The medical management of migraine. Am J Ther. 2004;11(2):130-40.

30. Chowdhury D. Acute management of migraine. J Assoc Phys India. 2010;58(Suppl):21-5.

31. Diamond $\mathrm{M}$, Cady R. Initiating and optimizing acute therapy for migraine: the role of patientcentered stratified care. Am J Med. 2005;118(Suppl 1):18s-27s.

32. Marissa Lagman-Bartolome A, Lay C. The traffic light of headache: simplifying acute migraine management for physicians and patients using the Canadian Headache Society Guidelines. Headache. 2019;59(2):250-2.

33. Sculpher M, Millson D, Meddis D, Poole L. Costeffectiveness analysis of stratified versus stepped care strategies for acute treatment of migraine: the disability in strategies for care (DISC) study. Pharmacoeconomics. 2002;20(2):91-100. 
34. Belvis R, Mas N, Aceituno A. Migraine attack treatment: a tailor-made suit, not one size fits all. Recent Pat CNS Drug Discov. 2014;9(1):26-40.

35. Erlichson K, Waight J. Therapeutic applications for subcutaneous triptans in the acute treatment of migraine. Curr Med Res Opin. 2012;28(7):1231-8.

36. Rothrock JF, Morey V. "Mixing triptans": patient satisfaction. Headache. 2011;51(1):135-40.

37. Taylor FR. Migraine headache: options for acute treatment. Curr Neurol Neurosci Rep. 2005;5(2): 86-92.

38. Lipton RB, Fanning KM, Serrano D, Reed ML, Cady $\mathrm{R}$, Buse DC. Ineffective acute treatment of episodic migraine is associated with new-onset chronic migraine. Neurology. 2015;84(7):688-95.

39. Messali A, Sanderson JC, Blumenfeld AM, et al. Direct and indirect costs of chronic and episodic migraine in the United States: a web-based survey. Headache. 2016;56(2):306-22.

40. Lipton RB, Buse DC, Serrano D, Holland S, Reed ML. Examination of unmet treatment needs among persons with episodic migraine: results of the American Migraine Prevalence and Prevention (AMPP) study. Headache. 2013;53(8):1300-11.

41. Bonafede M, Cai Q, Cappell K, et al. Factors associated with direct health care costs among patients with migraine. J Manag Care Spec Pharm. 2017;23(11):1169-76.

42. Buse DC, Pearlman SH, Reed ML, Serrano D, NgMak DS, Lipton RB. Opioid use and dependence among persons with migraine: results of the AMPP study. Headache. 2012;52(1):18-36.
43. Fischer M, Frank F, Wille G, Klien S, Lackner P, Broessner G. Triptans for acute migraine headache: current experience with triptan use and prescription habits in a tertiary care headache outpatient clinic: an observational study. Headache. 2016;56(6):952-60.

44. Foley KA, Cady R, Martin V, et al. Treating early versus treating mild: timing of migraine prescription medications among patients with diagnosed migraine. Headache. 2005;45(5):538-45.

45. Landy SH, Turner IM, Runken MC, Lee M, Sulcs E, Bell CF. A cross-sectional survey to assess the migraineur's medication decision-making beliefs: determining when a migraine is triptan-worthy. Headache. 2013;53(7):1134-46.

46. Baron EP, Markowitz SY, Lettich A, et al. Triptan education and improving knowledge for optimal migraine treatment: an observational study. Headache. 2014;54(4):686-97.

47. Friedman BW, Solorzano C, Norton J, et al. A randomized controlled trial of a comprehensive migraine intervention prior to discharge from an emergency department. Acad Emerg Med. 2012;19(10):1151-7.

48. United States Census Bureau. Annual estimates of the resident population by single year of age and sex for the United States: April 1, 2010 to July 1, 2018 [internet]. https://factfinder.census.gov/faces/ tableservices/jsf/pages/productview.xhtml?src= bkmk.

49. Lipton RB, Bigal ME, Diamond M, Freitag F, Reed ML, Stewart WF. Migraine prevalence, disease burden, and the need for preventive therapy. Neurology. 2007;68(5):343. 